\title{
Nonlinear modeling and transition corridor calculation of a tiltrotor without cyclic pitch
}

\author{
Yourong Fan ${ }^{1, *}$, Xinhua Wang ${ }^{1}$, Zhe $\mathrm{Hu}^{2}$, and Kai Zhang ${ }^{2}$ \\ ${ }^{1}$ College of Automation Engineering, Nanhang University, Nanjing, China \\ ${ }^{2}$ China Ship Development and Design Center, Wuhan, China
}

\begin{abstract}
In order to solve the problem of rotor airflow interference to the wing of tiltrotor UAV, the lift and drag in the slipstream area and the free flow area were calculated respectively according to the hydrodynamics theory and CFD simulation. The longitudinal nonlinear dynamics model of tiltrotor UAV is established by Newton-Euler method. In order to solve the problem that the lift and thrust are difficult to balance the body gravity in the transition flight mode, a method for calculating the transition corridor of a tiltrotor UAV without cyclic pitch is proposed. The boundary of the transition corridor is restricted by the Angle of attack of the wing and the thrust of the rotor. Considering the requirements of UAV cruise speed, flight safety and minimum energy consumption, the optimal transition curve is selected. The result show that the designed transition curve can ensure that the lift and the rotor thrust can balance the gravity completely and the Angle of attack is in a reasonable range, and the rotor force has enough margin of safety.
\end{abstract}

Keywords: Tiltrotor UAV, Nonlinear modeling, Slipstream area, Transition corridor.

\section{Introduction}

Tiltrotor UAV has the advantages of long endurance and fast speed of fixed-wing UAV, it can hover and take off and land vertically like a helicopter, so it has a wide application prospect in various fields. The tiltrotor UAV described in this article is shown in Figure 1 and Figure 2. Four tilting rotor systems are mounted on the wing and one vertical rotor system is mounted between the fuselage and the tail; all the rotors are operated without cyclic pitch. There are many rotor systems on the wing, which will have a great impact on the aerodynamic force of the aircraft. Therefore, the influence of rotor flow should be considered when nonlinear modeling the aircraft [1]. When the four motors on the wing are tilted to the horizontal position, the UAV is in fixed wing mode. When they are tilted to the vertical position, the UAV is in copter mode. During the transition from copter mode to fixed wing mode, the gravity of the aircraft is balanced by the transition from the thrust of the rotor to the lift of the wing [2]. If the tilt angle is too large and the speed is too low, the resultant force of thrust and lift is difficult to balance the gravity of the aircraft, resulting in

\footnotetext{
*Corresponding author: fanyourong@nuaa.edu.cn
} 
a significant drop in the aircraft's altitude, which will seriously affect flight safety. Tilting transition can be completed only when the reasonable tilting angle is matched with the flight speed. Therefore, the calculation of appropriate nacelle angle and flight speed envelope is the key to successful transition [3].

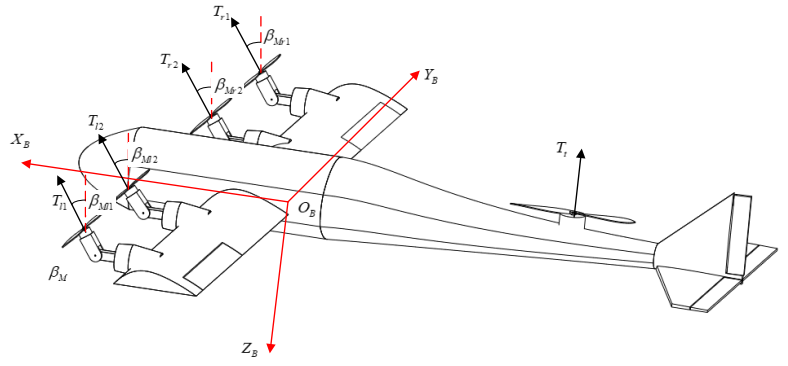

Fig. 1. Sample view of a tiltrotor UAV.

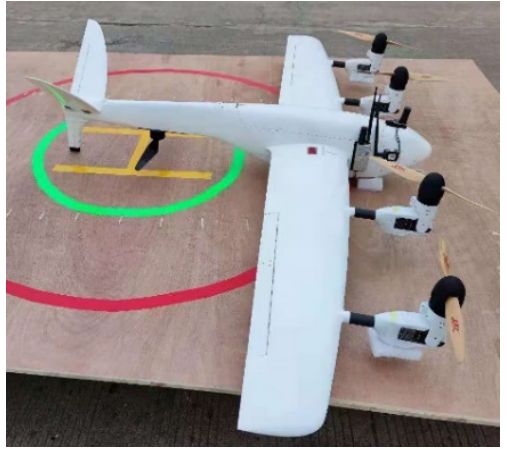

Fig. 2. Target tiltrotor UAV view.

\section{Longitudinal nonlinear model of a tiltrotor}

The body coordinate system is defined as shown in Figure 1, and the origin is on the center of gravity of the UAV. It is assumed that the UAV is symmetric in the XOZ plane of the body axis. During tilting, the changes of center of gravity and moment of inertia of the $\mathrm{UAV}$ are ignored, and the UAV is regarded as a rigid body. Based on the Newton-Euler method and the above assumptions, the longitudinal force and moment equations are derived, as shown in Equation (1)

$$
\left\{\begin{array}{l}
m(\dot{u}+w q-v r)=-m g \sin \theta+F_{A_{x}}+F_{T_{x}} \\
m(\dot{w}+v p-u q)=m g \cos \theta \cos \phi+F_{A_{z}}+F_{T_{z}} \\
\dot{q} I_{y y}+p r\left(I_{x x}-I_{z z}\right)+\left(p^{2}-r^{2}\right) I_{x z}=M_{A_{y}}+M_{T_{y}}
\end{array}\right.
$$

In the formula, $F_{T_{x}}, F_{T_{z}}$ and $M_{T_{y}}$ are the forces and moments caused by the thrust of the rotor and they can be calculated according to the relationship between the thrust and the nacelle angle and the geometric position of the rotor. The forces and moments $F_{A_{x}}, F_{A_{z}}$ and $M_{A y}$ are caused by air dynamics.

Assuming that the aircraft is flying without sideslip, the lift and drag are projected to the body coordinate system respectively, and the components of aerodynamic force in the aircraft system are obtained as follows:

$$
\left\{\begin{array}{l}
F_{A_{x}}=L \sin \alpha-D \cos \alpha \\
F_{A_{z}}=-L \cos \alpha-D \sin \alpha
\end{array}\right.
$$

During the flight, the airflow generated by multiple rotors in front of the wing will affect the aerodynamic characteristics of the wing. Therefore, in order to establish an accurate tiltrotor UAV model, it is necessary to consider the aerodynamic interference of the rotor airflow on the wing [4]. 


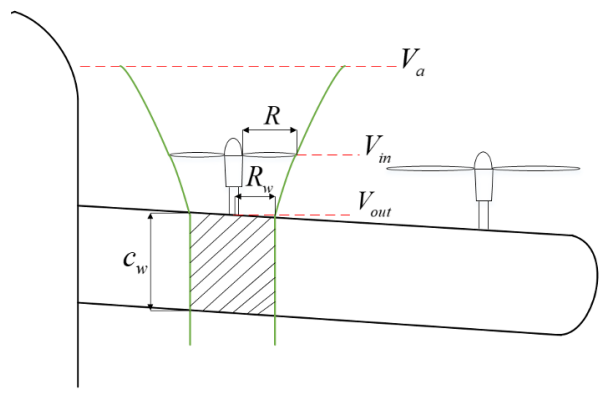

Fig. 3. The schematic diagram of slipstream area in fixed-wing mode.

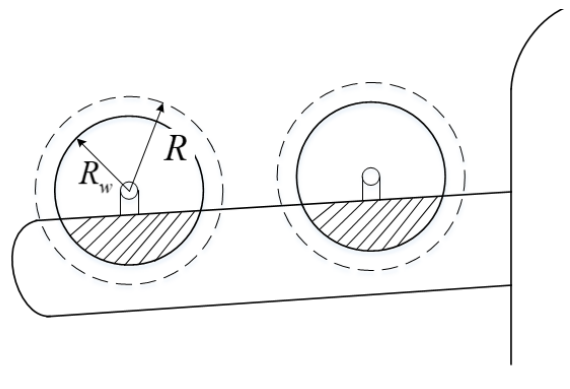

Fig. 4. The schematic diagram of slipstream area in copter mode.

In reference [5], numerical simulation was carried out based on the three-dimensional flow field, and the influence of slipstream on aerodynamic coefficient was quantitatively given. Reference [6] only considers the influence of rotor downwash airflow on the wing in helicopter mode, but does not consider the influence of rotor airflow on the wing after the nacelle tilts to the horizontal position. Reference [7] only considers the influence of propeller airflow on the wing in fixed wing mode. In this paper, the wing is divided into two parts: the slipstream area disturbed by rotor flow and the free flow area undisturbed. According to hydromechanics and rotor aerodynamics theory and CFD simulation, the area of the slipstream area and the relative airflow velocity in the whole tilting process were obtained, and the total lift, drag and pitching moment of the UAV are finally calculated. The contraction radius [8] of rotor wake on the wing is Equation

$$
R_{w}=R\left(0.78+0.22 e^{\left(-0.3-2 L_{n} \sqrt{C_{T}}-60 C_{T}\right)}\right)
$$

where $L_{n}$ is the length of the nacelle, $R$ is the radius of the rotor and $C_{T}$ is the drag coefficient of the rotor.

The slipstream area when the nacelle is vertical to the fuselage is shown by the shadow in Figure 4, and its area can be calculated by the grid method. the slipstream area when the nacelle is parallel to the fuselage is shown by the shadow in Figure 3 and its area is $S_{s s}=$ $2 R_{w} c_{w}$. According to the CFD simulation, the areas of the slipstream area on the wing are all $2 R_{w} c_{w}$ when the nacelle angle is greater than $60^{\circ}$, and the area of the slipstream area on the wing has an approximately linear relationship with the nacelle angle. The area of the slipstream area is given by (4)

$$
S_{s s}=\left\{\begin{array}{l}
S_{s s 0}+\frac{2 R_{w} c_{w}-S_{s s 0}}{60} \beta_{M}, 0 \leq \beta_{M}<60^{\circ} \\
2 R_{w} c_{w}, \beta_{M} \geq 60^{\circ}
\end{array}\right.
$$

The relative flow velocity relationship of the slipstream area is shown in Figure 3, where $V_{a}$ is the airspeed of the UAV, $V_{\text {in }}$ is the air velocity at the propeller, $V_{\text {out }}$ is the air velocity at the wing. According to the reference [9] and the aircraft angle of attack and the nacelle angle, the equations (5) is given. 


$$
\left\{\begin{array}{l}
m=\rho \pi V_{\text {in }} R_{w}{ }^{2} \\
T V_{\text {in }}=\frac{1}{2} m\left[V_{\text {out }}{ }^{2}-\left(V_{a} \cos \alpha \sin \beta_{M}\right)^{2}\right] \\
V_{\text {in }}=V_{a} \cos \alpha \sin \beta_{M}+v_{p} \\
V_{\text {out }}=V_{a} \cos \alpha \sin \beta_{M}+v_{s s}
\end{array}\right.
$$

where $\mathrm{T}$ is the thrust of one rotor, $\mathrm{m}$ is the air mass passing through any section of slipstream in unit time, $\alpha$ is the angle of attack of the UAV, and $\beta_{M}$ is the nacelle angle.

The induced velocity of the rotor on the wing $v_{s s}$ is obtained from the equations (5):

$$
v_{s s}=\sqrt{\frac{2 T}{\rho \pi R_{w}^{2}}+\left(V_{a} \cos \alpha \sin \beta_{M}\right)^{2}}-V_{a} \cos \alpha \sin \beta_{M}
$$

The airflow velocity in the slipstream area on the wing is obtained by the superposition of the induced velocity of the rotor on the wing and the flight airspeed [10]. The forward velocity and vertical velocity decomposed into the aircraft system are as follows:

$$
\left\{\begin{array}{l}
V_{x s s}=v_{s s} \sin \beta_{M}+V_{a} \cos \alpha \\
V_{z s s}=v_{s s} \cos \beta_{M}+V_{a} \sin \alpha
\end{array}\right.
$$

Without considering the sideslip angle, the angle of attack at the slipstream area $\alpha_{s S}$ is:

$$
\alpha_{s s}=\arctan \frac{\left(v_{s s} \cos \beta_{M}+V_{a} \sin \alpha\right)}{\left(v_{s s} \sin \beta_{M}+V_{a} \cos \alpha\right)}
$$

The central airspeed of the slipstream area $V_{\text {ass }}$ is:

$$
V_{a s s}=\sqrt{V_{x s s}^{2}+V_{z s s}^{2}}
$$

Based on the above derivation, the longitudinal aerodynamic force and moment on the wing can be expressed as:

$$
\left\{\begin{array}{l}
L=\frac{1}{2} \rho V_{a s s}^{2} C_{L}\left(\alpha_{s s}\right) 4 S_{s s}+\frac{1}{2} \rho V_{a}^{2} C_{L}(\alpha)\left(S-4 S_{s s}\right) \\
D=\frac{1}{2} \rho V_{a s s}^{2} C_{D}\left(\alpha_{s s}\right) 4 S_{s s}+\frac{1}{2} \rho V_{a}^{2} C_{D}(\alpha)\left(S-4 S_{s s}\right) \\
M_{A_{y}}=\frac{1}{2} \rho V_{a s s}^{2} C_{m}\left(\alpha_{s s}\right) 4 S_{s s} c+\frac{1}{2} \rho V_{a}^{2} C_{m}(\alpha) 4\left(S-4 S_{s s}\right) c
\end{array}\right.
$$

where, $C_{L}, C_{D}$ and $C_{m}$ are the functions of the lift coefficient, drag coefficient and pitching moment coefficient with respect to the angle of attack respectively.

\section{Solution of tilting transition corridor}

In order to simplify the calculation, it is assumed that the four rotors in front of the wing have the same thrust and the same nacelle angle. During the transitional flight, there is no obvious change in attitude and altitude of the tiltrotor UAV, and the acceleration is approximately zero [11]. The resultant force and moment acting on it are equal to zero, so $q=0, \dot{u}=\dot{w}=0, \beta=0$. Ignore the influence of the rudder surface and external disturbances. The longitudinal motion equation of the aircraft is (11): 


$$
\left\{\begin{array}{l}
4 T \sin \beta_{M}+L \sin \alpha-D \cos \alpha-m g \sin \theta=0 \\
-4 T \cos \beta_{M}-T_{t}-L \cos \alpha-D \sin \alpha+m g \cos \theta=0 \\
4 T \cos \beta_{M} d x_{1}-T_{t} d x_{2}+M_{y}=0
\end{array}\right.
$$

During the tilting process, the gravity of UAV is balanced by the lift and thrust. The lift is constrained by the airspeed and the angle of attack of the aircraft, and the thrust is constrained by the nacelle angle and the maximum thrust provided by the rotor. Based on the above constraints, the tilting transition corridor is divided into a high-speed section and a low-speed section [12].

\subsection{Angle of attack constraint}

When the angle of attack of the aircraft is greater than the stall angle of attack, the lift of the aircraft drops sharply, which brings danger, so the angle of attack in the process of tilting transition is less than the stall angle of attack $\alpha_{\text {stalling }}$. When the tiltrotor is flying forward in the copter mode, if the pitching angle is too large, the wing is equivalent to a huge drag plate, which causes a lot of energy consumption, so the angle of attack in the tilting process is greater than the zero-lift angle of attack $\alpha_{0}$. The angle of attack constraint is given as follows:

$$
\alpha_{0}<\alpha \leq \alpha_{\text {stalling }}
$$

\subsection{Rotor thrust constraint}

The thrust of target UAV is not controlled by cyclic pitch, but by changing the RPM, which is different from the traditional helicopter. Therefore, when calculating the transition corridor, it is not necessary to establish a power model as complex as [13]. We only need to get the thrust of the rotor through a simple RPM relationship. The front and tail rotor systems of the target UAV use different motors and blades to balance the pitching moment, and the maximum value of the rotor's thrust is different. The required thrust during the tilting transition should be less than $65 \%$ of the maximum thrust to reserve enough control margin against unknown external interference. Therefore, the thrust constraint of the rotor is (13).

$$
\begin{aligned}
& T_{\text {front }} \leq 65 \% \max \left(T_{\text {front }}\right) \\
& T_{\text {tail }} \leq 65 \% \max \left(T_{\text {tail }}\right)
\end{aligned}
$$

In the formula, the maximum thrust of the front rotor $\max \left(T_{\text {front }}\right)$ and the maximum thrust of the rear rotor $\max \left(T_{\text {tail }}\right)$ are determined by the selection of the motor and the propeller.

\section{Experiment results}

The target UAV is shown in Figure 2. The matching motors and propellers are selected so that the UAV has enough control margin in the copter mode. The thrust of the front and rear rotor systems changes with the rotation speed as shown in Figure 5. 

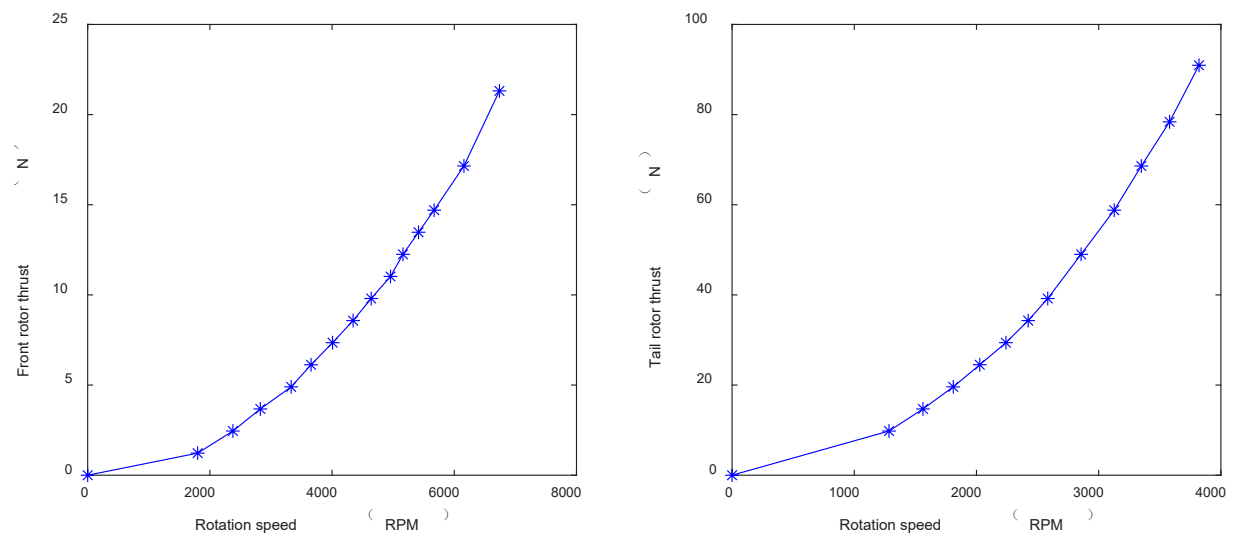

Fig. 5. The relationship between rotor thrust and RPM.

The aerodynamic force and moment coefficients of the aircraft are calculated by CFD simulation, and the lift coefficient and drag coefficient are shown in Figure 6.
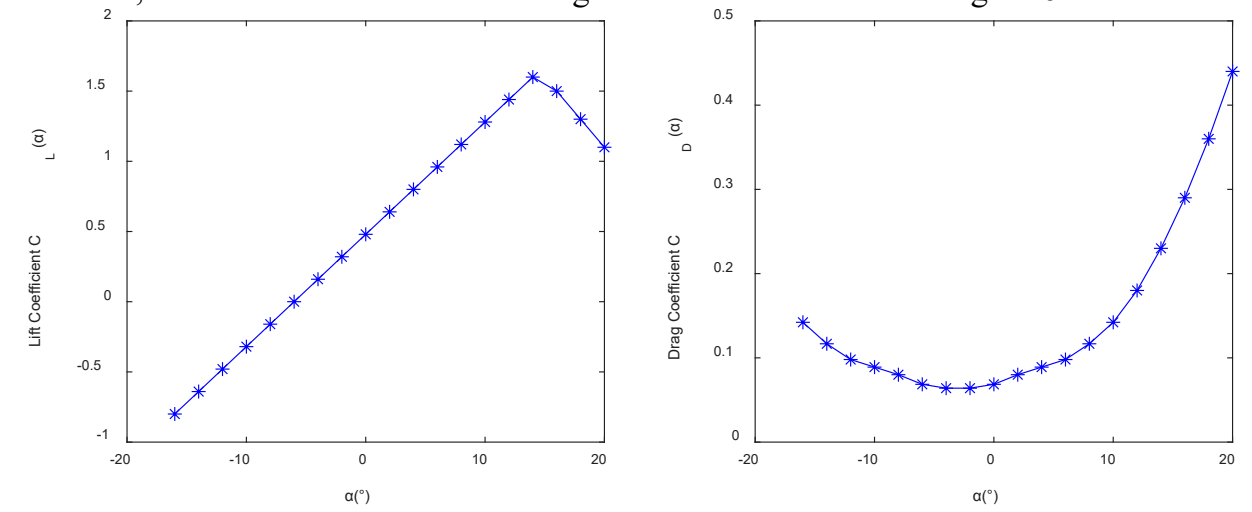

Fig. 6. Aerodynamic characteristic curve of the target UAV.

The constraints in equations (12) and (13) can be obtained according to the aerodynamic characteristics of the wing and rotor thrust characteristics. Combined with the longitudinal simplified equation (11) and the aerodynamic force and moment calculation method described in part 2, the envelope of the tilting transition corridor is calculated ,as shown by the solid line in Figure 7.

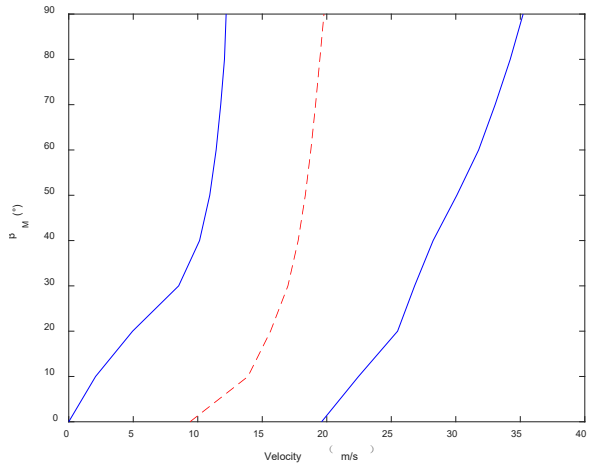

Fig. 7. Transition corridor curve of the target UAV. 
Select a transition curve in the envelope of the transition corridor. The selection of the curve requires:

(a). The pull of the rotor has sufficient margin;

(b). The angle of attack of is within a safe range and the drag is small, which reduces the battery power consumption;

(c). The speed corresponding to the end of the transition curve is the cruise speed of the aircraft.

According to the above selection rules, the starting point of the designed transition curve corresponds to the nacelle angle of $0^{\circ}$, and the speed is $9.4 \mathrm{~m} / \mathrm{s}$. The end point of the designed transition curve corresponds to the nacelle angle of $90^{\circ}$, and the speed is the cruise speed of the UAV $19.8 \mathrm{~m} / \mathrm{s}$. Between the starting point and the end point, a working point is selected every $10^{\circ}$ at the nacelle Angle. Set the corresponding angle of attack to calculate the trim speed. Some data are shown in Table 1. Tilt transition curve is drawn according to Table 1, as shown by the dotted line in Figure 7.

Table 1. Formatting sections, subsections and subsubsections.

\begin{tabular}{ccc} 
Nacelle angle $(\mathrm{deg})$ & Velocity $\left(\mathrm{ms}^{-1}\right)$ & angle of attack $(\mathrm{deg})$ \\
\hline 0 & 9.4 & -1.5 \\
10 & 13.9 & 0 \\
20 & 15.6 & 1 \\
40 & 17.8 & 2 \\
60 & 18.8 & 2 \\
70 & 19.1 & 2 \\
90 & 19.8 & 2 \\
\hline
\end{tabular}

The curves of rotor thrust, body lift and drag with nacelle angle corresponding to the designed transition curve are shown in Figure 8. It can be seen from the curve that the lift increases with the increase of nacelle angle, and finally balances with the gravity of UAV. The rotor thrust gradually decreases with the increase of the nacelle angle, and finally becomes zero. During the whole transition process, the drag of the tiltrotor UAV is small, the lift drag ratio is large, and the angle of attack is kept in a reasonable range.

Figure 9 is the curve of the front and tai rotors' thrust with the nacelle angle. From the curve, it can be seen that the thrust value of each rotor decreases with the increase of the nacelle angle, and the maximum value of the pull meets the requirements of equation (13)

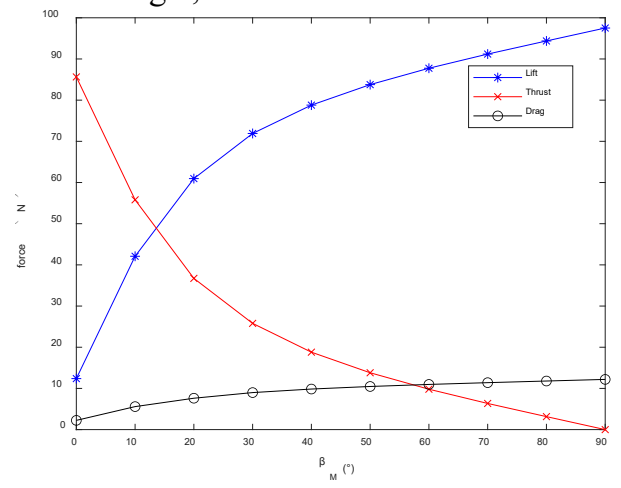

Fig. 8. Change curve of lift, drag and rotor thrust with nacelle angle.

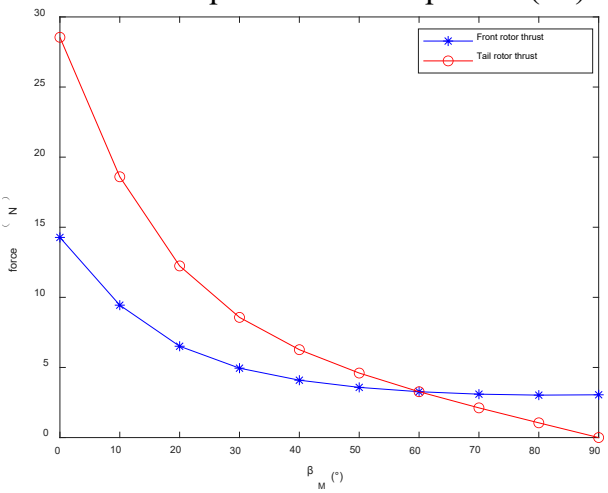

Fig. 9. Change curve of the front and rear rotor thrust with nacelle angle. 


\section{Conclusion}

In this paper, the calculation model of slipstream area and relative airspeed is derived by fully considering the interference of rotor flow on the wing. A longitudinal nonlinear model of a tiltrotor UAV without cyclic pitch was established by Newton-Euler method. Combined with practical engineering constraints and longitudinal trim simplification, the tilting transition corridor of UAV is calculated. According to the actual flight requirements, a transition curve is designed in the transition corridor, and the correctness of it is verified by simulation calculation. The transition curve designed in this paper can provide a reference for the attitude and speed control in the transition process.

\section{References}

1. Francesco, G. D. , and M. Mattei. Modeling and Incremental Nonlinear Dynamic Inversion Control of a Novel Unmanned Tiltrotor. Journal of Aircraft 53.1(2016):1-14.

2. Wei, et al. Robust transition control of a Martian coaxial tiltrotor aerobot. Acta Astronautica 99.1(2014):111-129. Wei, et al.

3. Chao, C. , et al. Mathematical modeling and control of a tiltrotor UAV. IEEE International Conference on Information \& Automation IEEE, 2017.

4. Li ZQ, Xia PQ. Aeroelastic stability of full-span tiltrotor aircraft model in forward flight. Chin J Aeronaut 2017;30(6):1885-94.

5. CHEN H, LU ZL, GUO TQ. Rotor slipstream effects on aerodynamic performance of tiltrotor aircraft in airplane mode. Journal of Aerospace Power 2018,33(11):2809-2816 [Chinese].

6. Polak, D. R. , and A. R. George. Flowfield and Acoustic Measurements from a Model Tiltrotor in Hover. Journal of Aircraft (1998).

7. Yuksek, B., et al. Transition Flight Modeling of a Fixed-Wing VTOL UAV. Journal of Intelligent \& Robotic Systems 84.1-4(2016):1-23.

8. Landgrebe, Anton J. An Analytical and Experimental Investigation of Helicopter Rotor Hover Performance and Wake Geometry Characteristics. (1971).

9. Steijl, R. , and G. Barakos. Sliding mesh algorithm for CFD analysis of helicopter rotor-fuselage aerodynamics. International Journal for Numerical Methods in Fluids 58.5(2010):527-549.

10. Zhiquan L I , Xia P, Engineering C , et al. Aeroelastic stability of full-span tiltrotor aircraft model in forward flight[J]. Chinese Journal of Aeronautics, 2017.

11. Garcia, A. J., and G. N. Barakos. Numerical simulations on the ERICA tiltrotor. Aerospace Science \& Technology 64.MAY(2017):171-191.

12. Hernandez-Garcia, R. G. , and H. Rodriguez-Cortes . Transition flight control of a cyclic tiltrotor UAV based on the Gain-Scheduling strategy. IEEE(2015):951-956.

13. Papachristos, C. , K. Alexis, and A. Tzes. Design and experimental attitude control of an unmanned Tilt-Rotor aerial vehicle. International Conference on Advanced Robotics IEEE, 2011. 